\title{
Design OF THE Prototype of A Full ARM MECHATRONICS REHABILITATION DEVICE
}

\author{
Tea Arrigoni, ${ }^{1,2}$ Saša Zelenika,,${ }^{1,2}$ Ervin Kamenar ${ }^{1,2}$ \& \\ Tea Schnurrer Luke Vrbanić ${ }^{3}$ \\ ${ }^{1}$ University of Rijeka, Faculty of Engineering, Vukovarska 58, 51000 Rijeka, Croatia \\ ${ }^{2}$ University of Rijeka, Centre for Micro- and Nanosciences and Technologies, \\ Radmile Matejčić 2, 51000 Rijeka, Croatia \\ ${ }^{1}$ University of Rijeka, School of Medicine, Braće Branchetta 20, 51000 Rijeka, Croatia \\ * Corresponding author: sasa.zelenika@ riteh.hr
}
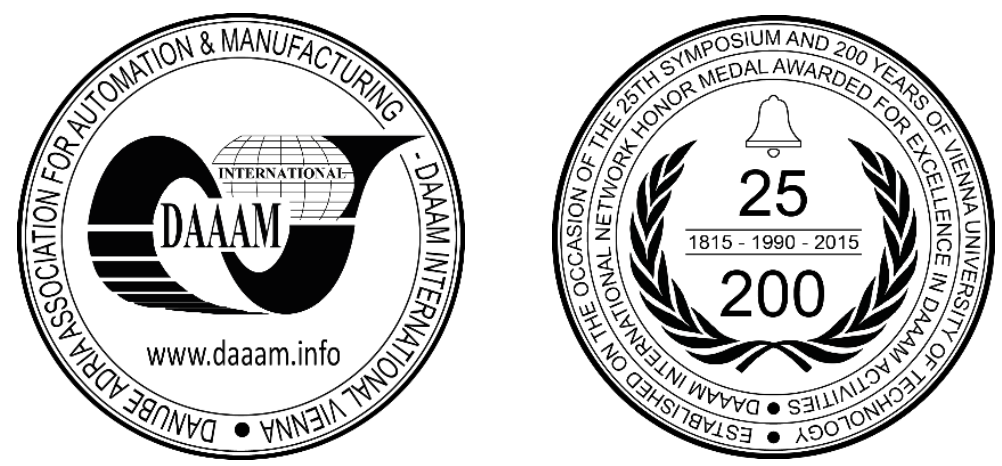

This Publication has to be referred as: Arrigoni, T[ea]; Zelenika, S[asa]; Kamenar, E[rvin] \& Schnurrer Luke Vrbanic, T[ea] (2018). Design of the Prototype of a Full Arm Mechatronics Rehabilitation Device, Proceedings of the 29th DAAAM International Symposium, pp.0016-0024, B. Katalinic (Ed.), Published by DAAAM International, ISBN 9783-902734-20-4, ISSN 1726-9679, Vienna, Austria

DOI: $10.2507 / 29$ th.daaam.proceedings.003

\begin{abstract}
Physical rehabilitation therapy is the proven method of regaining the functionality of impaired human arm movements, especially those resulting from stroke - the leading cause of adult disability in western countries. Traditional therapy requires physical therapists whose limited number and time availability restricts the rehabilitation outcomes. Mechatronics-based rehabilitation devices, which adaptively assist human movements, are nowadays a viable solution to cope with these shortcomings. The commercially available devices are, however, expensive and capacious, while their adaptability to the rehabilitation of both arms is limited. A crucial task in designing complex rehabilitation devices, which allow overcoming these difficulties, is the study of the kinematics of arms' movements. It is postulated that a structure with seven degrees-of-freedom can assure a good combination of motion accuracy and acceptable device complexity. Electronics components (actuators with their drivers and power supplies as well as the feedback sensors) fitting the characteristics of the resulting mechanism must hence be chosen, and an appropriate simultaneous real-time control system is to be developed. Based on these considerations, the design of a prototype of a versatile, portable, efficient and cost-effective full arm rehabilitation device, providing high intensity and frequency treatment of both arms, is presented in this work.
\end{abstract}

Keywords: rehabilitation; mechatronics; mechatronics device design 


\section{Introduction}

Stroke is a neurological disease with the highest prevalence (each year more than 17 million people worldwide suffer from stroke) and it is the leading cause of disability in western countries [1]. One of the major consequences of stroke is acute hemiparesis that affects the upper limbs and that, in addition to stroke, can be induced also by congenital causes, muscular dystrophy or atrophy, spinal cord injuries or cerebral palsies [2]. Studies have shown that, in order to improve the respective recovery outcomes, rehabilitation therapy has to be initiated as soon as patients' health conditions allow it. The rehabilitation process is in any case generally long-term and, while several patients fully recover, some of them need assistance throughout the remaining life [2].

Traditional therapy is performed with the assistance of physiotherapists, where each therapist is committed to one patient at a time. It is obvious that the limited number and time availability of therapists can limit in this case the rehabilitation process to a period shorter than required. What is more, the traditional therapy sessions do not allow an adequate training repeatability nor an objective measurement of the achieved progress in the recovery process, limiting therefore also patients' motivation for further rehabilitation [3]. Mechatronics-based rehabilitation devices, which adaptively assist human movements, are nowadays a viable solution to cope with these shortcomings [2]. The commercially available devices are, however, expensive and capacious, while their adaptability to the rehabilitation of all arm's movements or of both arms is limited.

In recent literature it was shown that a rehabilitation structure with a sufficient number of active degrees-of-freedom (DOFs) can lead to a satisfactory motion accuracy with an acceptable device complexity [2]. Electronics components (actuators with drivers and power supplies and the feedback sensors) fitting the characteristics of the resulting mechanism must hence be chosen, and an appropriate simultaneous real-time control system is to be developed.

The aim of this work is thus to design the prototype of a full arm mechatronics rehabilitation device with all its electromechanical components. The aspects taken into consideration in this framework are: cost effectiveness, versatility of use, portability, simple maintenance and user-friendly set-up and control.

The basis of human arm anatomy, needed in the development of the mechatronics rehabilitation device is hence described in section 2 of this paper. Based on the analysis of the state-of-the-art rehabilitation devices performed in section 3 , the design of the herein proposed original device, obtained in the CATIA ${ }^{\circledR}$ V5 CAD software, complemented with the selection of the corresponding actuators, sensors and the control hardware, is proposed in section 4 . The conclusions and an outlook for future work, given in section 5, sum-up, finally, the performed work.

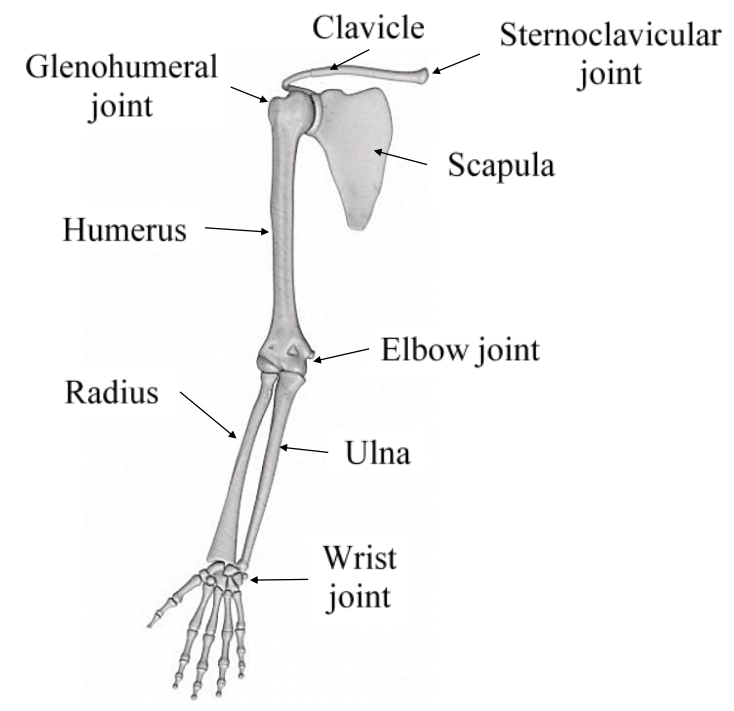

Fig. 1. Human arm anatomy

\section{Basis of human arm anatomy}

In modelling the rehabilitation device, it is important that its kinematics reproduces as much as possible that of the human arm itself, while concurrently guaranteeing the maximal simplicity of the resulting design. On the other hand, if the design of the rehabilitation device is over-simplified, it could lead to misalignments between the device and patient's arm, with a potential negative impact on the rehabilitation. A wide-ranging communication and established collaboration with the Clinical Hospital Centre in Rijeka, Croatia [4], and especially with their department of Physical and Rehabilitation Medicine, is thus carried on to exchange the information regarding their best practices and make use of their expertise gained through the work with a large number of patients. Based on this information, a study of the anatomy of the human arm is summarised in Figure 1. It can thus be seen that the upper arm, made of the longest arm's bone, the humerus, is joined in the elbow with the lower arm (or forearm), constituted by two parallel bones - the ulna and the radius. 
The three mentioned bones are the basic segments of the human arm whose length is extremely important for the development of the rehabilitation device. The shoulder, articulated by the humerus and the cavity (the "socket") of the scapula, held together by ligaments, forms the glenohumeral joint that provides a wide range of motions coupled, via the clavicle and the scapula, to the movements of the whole shoulder girdle. The shoulder has, in fact, five interacting DOFs but, since some of them introduce partial constraints to the other ones, it can be approximated quite accurately by a combination of a joint with three-rotational DOFs (i.e. a spherical joint) with corresponding leverages between them. If the full range of the upper-body movements has to be achieved, the three shoulder DOFs must then be synergistically actuated. The elbow is, in turn, a one DOF revolute joint that permits the flexion and extension of the forearm, while allowing an additional DOF in the form of a twist of the forearm with respect to the ulna. The radius bone articulates with the bones forming the major joint of the wrist that provides itself two additional rotations with the respective axes intersecting in the wrist joint. The wrist can thus be assimilated to a universal Cardan joint [5], [6].

All these facts reduce, thus, to the quest that the joints of the mechatronics device approximate as much as possible the motion constraints of the shoulder, the elbow and the wrist [7]. While for the elbow this is rather straightforward, in the case of the shoulder the undertaking becomes much more complex, especially considering the need to actuate the movements of the shoulder complex through its full range without causing any discomfort to the user [5]. The mechanical structure between the joints has, in turn, to be adjustable to the different lengths of the human arm in the ranges given in Table $1[6],[8]$.

\begin{tabular}{|c|c|c|c|c|}
\hline Part of human hand & Gender & Average dimension $(\mathbf{c m})$ & min. $(\mathbf{c m})$ & max. $(\mathbf{c m})$ \\
\hline \multirow{2}{*}{ Upper arm } & Male & 36.90 & 29.70 & 44.60 \\
\cline { 2 - 5 } & Female & 33.58 & 28.20 & 40.10 \\
\hline \multirow{2}{*}{ Lower arm } & Male & 26.99 & 21.20 & 32.50 \\
\cline { 2 - 5 } & Female & 24.34 & 15.70 & 31.20 \\
\hline
\end{tabular}

Table 1. Average dimensions of the human arm

\section{Critical analysis of available rehabilitation devices}

The rehabilitation devices available on the market can be divided into two main categories: passive and active devices. Passive devices are those without actuated joints that can thus not be used for the rehabilitation of patients with more severe motion disabilities. Passive devices are therefore generally used only for strengthening partially weakened patients' muscles. On the other hand, the idea of active rehabilitation devices is to stimulate the patients to use as much as possible their own efforts while adding to that, via actuated joints, the forces necessary to perform the required movements. The resulting motions can also be repeated at will at a determined frequency and with the needed intensity. The introduction of active devices to the rehabilitation process enables, thus, to increase the number of patients that can be concurrently handled by each physiotherapist to three or even more. An implicit outcome is that the patients get the needed therapy faster and with an increased number of training sessions, which leads also to a decrease of the duration of the recovery process. What is more, the typical values allowing an objective assessment of the rehabilitation process can be recorded for every patient, which not only provides a track-record of the achieved improvements, but also increases the motivation of the patients during the rehabilitation - thus enhancing further the positive outcomes of the rehabilitation process [2].

On the other hand, however, it is quite challenging to design active devices able to provide task-oriented movements that mimic daily activities such as eating, drinking, showering, dressing etc. In fact, with the aim of improving the motion functionality of patients' arms, the rehabilitation device has to allow specific exercises not only to build up the strength of the muscles, but also to provide a large motion range so as to increase the achievable (lost) range of motion. This can, however, be generally achieved only by increasing the number of DOFs at the expense of an increased complexity of the attained mechanism and of the respective control algorithms. What is more, the needed actuating power often implies the usage of large and heavy actuators that, in turn, due to their dimensions, limit the angular displacement and the workspace. The resulting state-of-the-art prototype mechatronics rehabilitation devices, providing a wide variety of design configurations, with differing numbers of DOFs, have thus the disadvantage of being heavy, bulky and very expensive (up to several hundreds of thousands of euros). What is more, they often do not provide the possibility of wrist rehabilitation [2], [9] [10], [11], [12].

\section{Design of the prototype of a full arm rehabilitation device}

Based on the performed study of the human arm anatomy and of the nowadays-available rehabilitation devices, it is concluded that a mechatronics configuration with seven active DOFs should provide a good combination of motion accuracy with an acceptable complexity. Such a configuration is thus chosen for the design of the active full arm rehabilitation device being developed in this work. What is more, the goals set for the device comprise also:

- the necessity to reduce the price so as to make the device affordable for hospital and rehabilitation clinics in Croatia;

- the need to make the dimensions of the device compact (ideally the device should be portable) but also adaptable to the needs of the patients and the physiotherapists, in terms of the dimensions (cf. Table 1) as well as of the adaptability of the provided power and the versatility to use the device for both arms; 
- the need to make the design of the device and its control as simple as possible while providing the necessary intensity, duration and frequency of the desired movements;

- the possibility to implement a patients' (users') interface that would motivate them for an active rehabilitation.

Based in these considerations, the 3D modelling of the innovative device proposed in this work is performed by using the CATIA ${ }^{\circledR}$ V5 CAD software (Figure 2). The supporting part of device is hence developed so that it is positioned behind patient's back and adjustable in height (Figure 2a). At the top of this column, two levers enable the conformation of shoulder's mechanical structure so as to attain a close resemblance with the anatomy and motion of the respective human joint. The three active rotational DOFs of the shoulder are then positioned to make them corresponding to those of the human arm (Figure 2b). The first actuated DOF of the shoulder complex enables therefore the abduction and adduction of the shoulder in the range of angles defined in Table 2. The respective actuator with its angular encoder is positioned at the backside, close to the scapula. The second active shoulder DOF enables the internal and external rotation with respect to the axis positioned above the shoulder itself. The third actuated DOF enables, in turn, the extension and flexion shoulder movements. It has to be noted here that the three axes of the designed shoulder complex intersect in a single point positioned at the centre of the human shoulder, thus enabling movements approximating closely the natural ones.

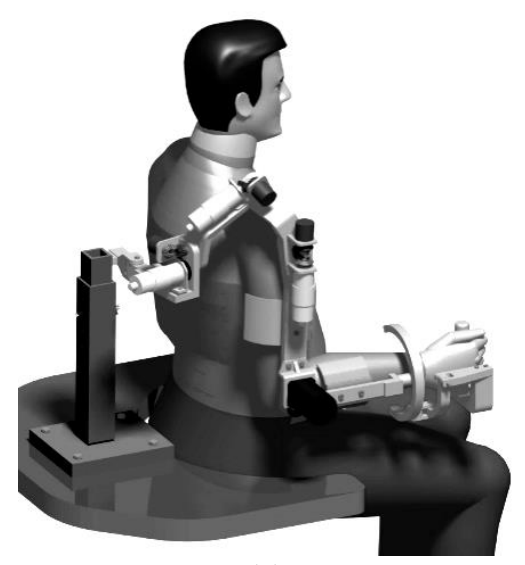

(a)

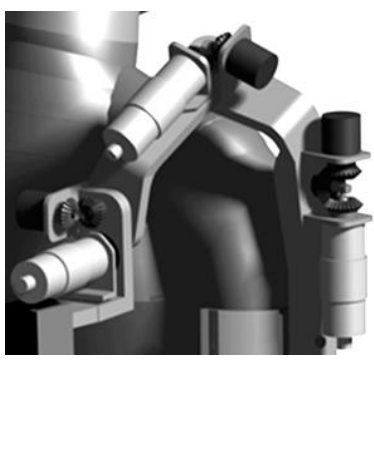

(b)
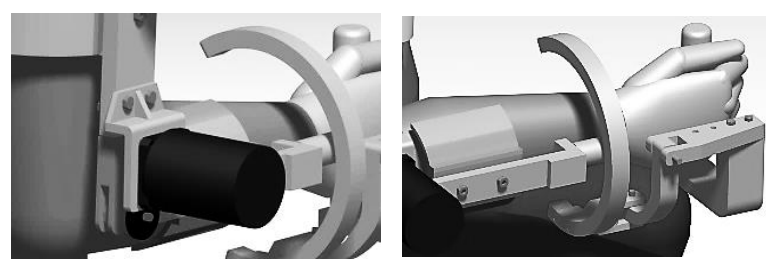

(c) (d)

Fig. 2. Design of the prototype of an innovative full arm mechatronics rehabilitation device (a) with details of the shoulder (b), elbow (c) and wrist (d) active joints

The extension and flexion DOF of the elbow as well as forearm's twisting DOF are assured by employing again servo actuators with feedback based on encoder signals (Figure 2c); the same is true also for the two rotational wrist DOFs whose axes intersect in a single point in the middle of the human wrist (Figure 2d). The twisting motion of the forearm (mimicking the overlap of the ulna and the radius bones) is assured in this frame coupled with the rotation movements of the wrist. The passive mechanical elements between the shoulder and the elbow and between the elbow and the wrist are, finally, designed so that they can be axially extended to enable the adaptation of their length to the dimensions of patients' hand. Both elements are also equipped with comfortable but firm cuffs ("wristbands") that connect the rehabilitation device to patients' arm.

All the motorised joints in the described design configuration are actuated via independent actuators enabling the torque of each joint to be controlled separately [13]. Based on recent literature [13], the respective actuators are chosen so that they can provide torques that correspond in value to the average torques produced by the human arm. It is to be noted that human movements have rather large velocity and acceleration peaks, thus implying a high level of dynamic interaction. The choice of the actuators has thus to be based on two contrasting requirements: on one hand smaller, lighter actuators with gearboxes can generate sufficient forces while reducing the overall size of the device but, on the other hand, gearboxes add friction to the system and potentially reduce the overall dynamics performances. Still, the advantages of DC motors in terms of their size, ease of control, efficiency, flexibility and price clearly outperform the option of eventually using pneumatic or hydraulic actuators that would provide high powers and response velocities with the pronounced drawbacks related to the increase in price, the complexity and the overall size of the resulting apparatus. On the other hand, considerations regarding the dimensions, price and the acquired expertise of the project team have directed the choice of the respective feedback sensors towards rotary encoders.

Based on these and on considerations relative to the needed forces, torques, velocities and motion ranges, all three actuators used in the shoulder complex of the developed device are chosen to be $25 \mathrm{Nm}$ DC motors (Table 2). Independent quadrature encoders with a $0.2^{\circ}$ resolution are used as feedback sensors that provide information about the angular displacement. The extension of the elbow is, in turn, achieved by employing a servo actuator with an embedded encoder, whereas the rotational wrist DOFs are driven by employing Faulhaber miniature actuators that can provide torques of up to $3.4 \mathrm{Nm}$ accompanied, again, by encoders with a $0.2^{\circ}$ resolution. The minimisation of the number of differing actuators has, obviously, advantages in terms of the development of the respective controllers. 
Each actuator is supplemented also with a current sensor used to quantify the produced torque. This enables that the value of the torques provided by the actuators is adaptively and precisely added to those that the patients can produce on their own, i.e., that the total forces match those necessary to perform the required movements without perturbing them. The resulting motions can also be repeated at will at a determined frequency and with the needed intensity. What is more, muscle sensors are foreseen to provide a signal of the will of the patient to initialise the motion. The issue of hyperextension, i.e., the avoidance of possible "crashes" with the device itself or the collision with patient's body, are, finally, avoided by using mechanical limits ("hard stops") as well as two electro-mechanical limit switches per joint. The thus chosen and purchased actuating and sensing elements are shown in Figure 3.

\begin{tabular}{|c|c|c|c|c|c|c|}
\hline Joint & Actuator & $\begin{array}{c}\text { Torque, } \\
\text { Nm }\end{array}$ & $\begin{array}{c}\text { Arm's } \\
\text { movement }\end{array}$ & Angle, ${ }^{\circ}$ & Sensors & Encoder \\
\hline Shoulder 1 & \multirow{3}{*}{$\begin{array}{c}\text { RMCS-2005 - Mega } \\
\text { Torque Planetary DC } \\
\text { Geared Motor 250W } 100 \\
\text { RPM, 180:1 }\end{array}$} & \multirow{3}{*}{25} & $\begin{array}{c}\text { Flexion/ } \\
\text { Extension }\end{array}$ & $-60 \ldots 180$ & \multirow{7}{*}{$\begin{array}{l}\text { Current } \\
\text { sensor } \\
\text { modules } \\
20 \mathrm{~A}\end{array}$} & \multirow{3}{*}{$\begin{array}{c}\text { Rotary quadrature } \\
\text { encoders } \\
\text { 2500PPR/10000CPR }\end{array}$} \\
\hline Shoulder 2 & & & $\begin{array}{l}\text { Abduction/ } \\
\text { Adduction }\end{array}$ & $-90 \ldots 180$ & & \\
\hline Shoulder 3 & & & $\begin{array}{c}\text { Lateral / } \\
\text { medial } \\
\text { rotation }\end{array}$ & $30 \ldots 100$ & & \\
\hline Elbow & $\begin{array}{c}\text { RMCS - 2201 - High } \\
\text { Torque Encoder DC Servo } \\
\text { Motor 100 RPM, 1800:1 }\end{array}$ & 11 & $\begin{array}{l}\text { Flexion/ } \\
\text { Extension }\end{array}$ & $0 \ldots 140$ & & $\begin{array}{l}\text { Encoder included in } \\
\text { motor }\end{array}$ \\
\hline Forearm & $\begin{array}{c}\text { Faulhaber minimotor SA } \\
20 / 19.7: 1\end{array}$ & \multirow{3}{*}{3,4} & $\begin{array}{l}\text { Pronation/ } \\
\text { Supination }\end{array}$ & $-90 \ldots 90$ & & \multirow{3}{*}{$\begin{array}{c}\text { Rotary quadrature } \\
\text { encoders } \\
\text { 2500PPR/10000CPR }\end{array}$} \\
\hline Wrist 1 & \multirow{2}{*}{$\begin{array}{l}\text { Faulhaber minimotor SA } \\
\qquad 16 / 743: 1\end{array}$} & & $\begin{array}{c}\text { Flexion/ } \\
\text { Extension }\end{array}$ & $-80 \ldots 70$ & & \\
\hline Wrist 2 & & & $\begin{array}{l}\text { Abduction/ } \\
\text { Adduction }\end{array}$ & $-20 \ldots 30$ & & \\
\hline
\end{tabular}

Table 2. Main actuating and sensing components of the rehabilitation device under development

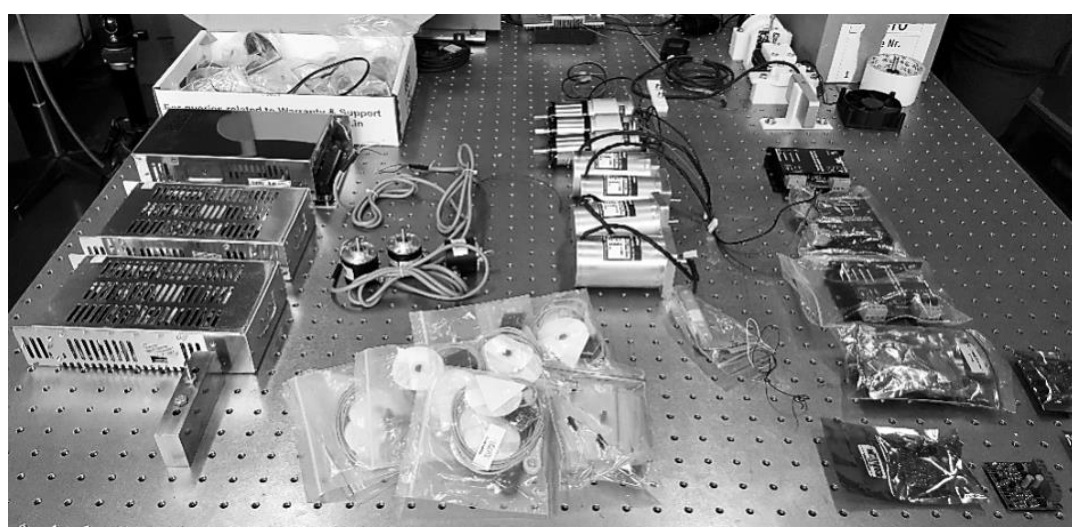

Fig. 3. Actuating and sensing components for the designed mechatronics rehabilitation device

In parallel to the design of the full arm mechatronics device, the suitable controllers are also developed and configured. The determination of a transformation map related to the trajectories of the joints' coordinates of the rehabilitation device, is thus performed. An inverse kinematics approach is followed [14] whereby, taking into account the real motion constraints of the shoulder, the elbow and the wrist of the human arm, the desired trajectory of the end effector of the rehabilitation device is transformed into the coordinates of the described seven DOFs of the joints of the device. A geometric approach based on the rotation matrices in Euclidean space and the Denavit-Hartenberg parameters, which allow simplifying the considered kinematics via coordinate transformations in a single homogeneous transformation matrix, is hence employed [15], [16]. Each homogeneous transformation is given in this frame by a product of four basic transformations associating the joints' coordinates to the respective links. The hence obtained coordinates of the joints are hence used as inputs for the design of the controllers of the rehabilitation device. The control hardware is based in this frame on the National Instruments MyRIO 1900, while the LabVIEW software, including the robotics module based on the Denavit-Hartenberg algorithms, is employed for the development of the control procedures. Each shoulder actuator is thus driven via an external DC motor driver corresponding to the chosen motor. RMCS-2302 drivers with pulse width modulation (PWM) outputs, whose duty cycle is controlled via a PID algorithm as a function of the distance to the reference position, as measured by the employed encoders, is hence used and controlled in the LabVIEW environment (Figure 4a). 
The elbow's extension actuator is, in turn, controlled via a PID controller that is embedded in its internal driver, while the communication with the control hardware is assured by employing asynchronous serial communication (i.e., the universal asynchronous receiver-transmitter - UART - Figure 4b). The remaining actuators are driven by using analogue power amplifiers and PID control algorithms.

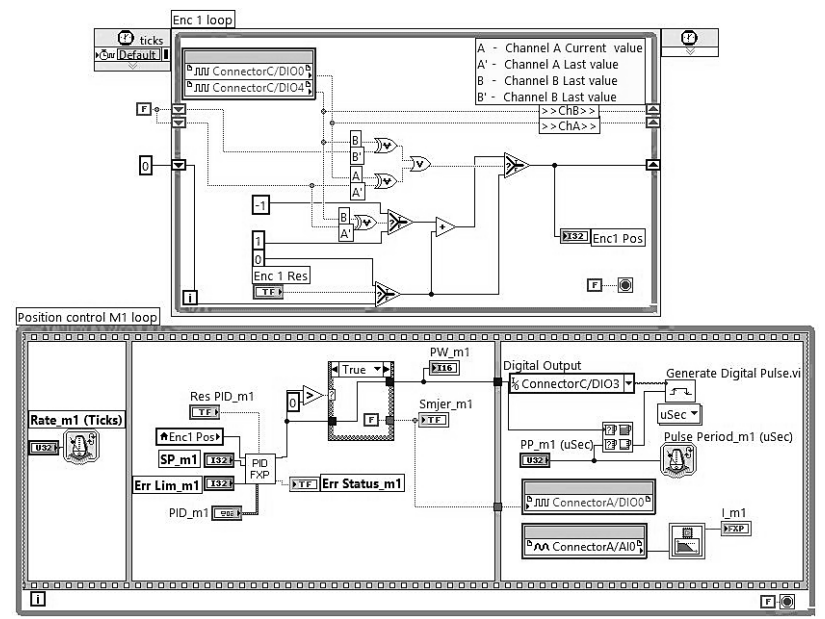

(a)

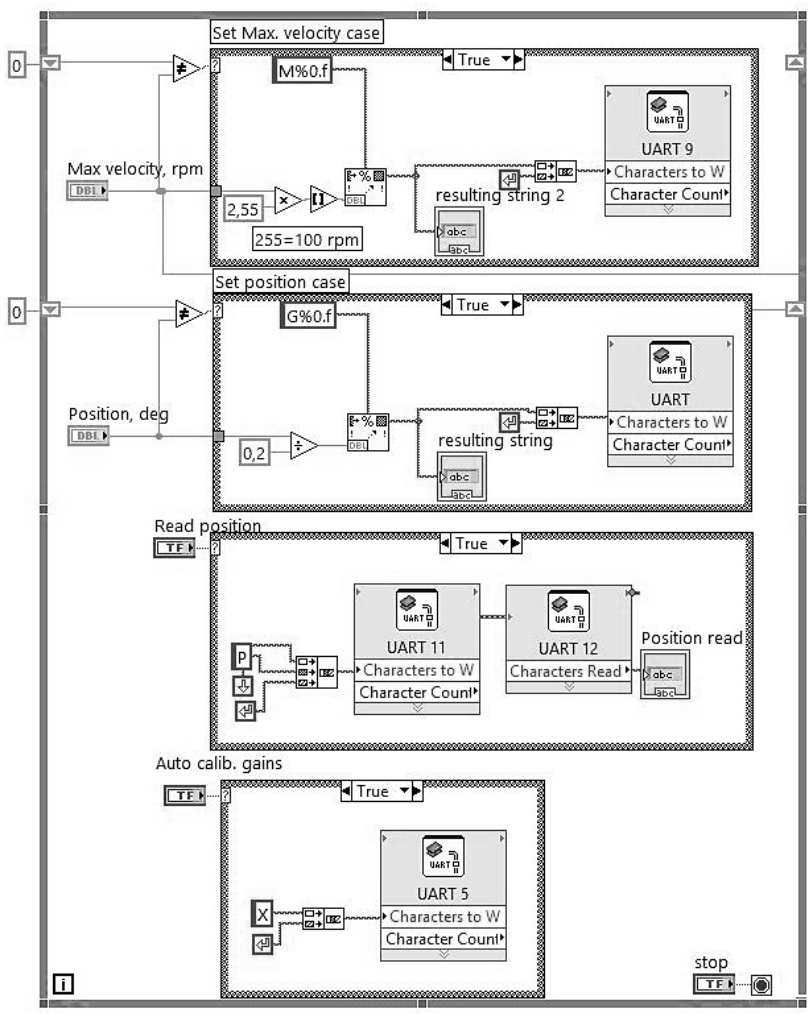

(b)

Fig. 4. LabVIEW Virtual Instruments for the control of the used actuators

\section{Material for the mechanical parts of the mechatronics device}

Based on the design of the full arm mechatronics rehabilitation device described in the previous section, it is estimated that most of the passive mechanical components, i.e., up to $60 \%$ of all the components of the device will be produced in the light yet strong acrylonitrile butadiene styrene (ABS) thermoplastic polymer by employing 3D printing. In fact, not only does this choice allow to attain the needed low-weight and versatility of the developed device [17], but also our research team has at its disposal, at the premises of the Centre for Micro- and Nanosciences and Technologies of the University of Rijeka, Croatia [18], a suitable Stratasys Fortus $250 \mathrm{mc} 3 \mathrm{D}$ printer based on the fused deposition modelling (FDM) technology [19].

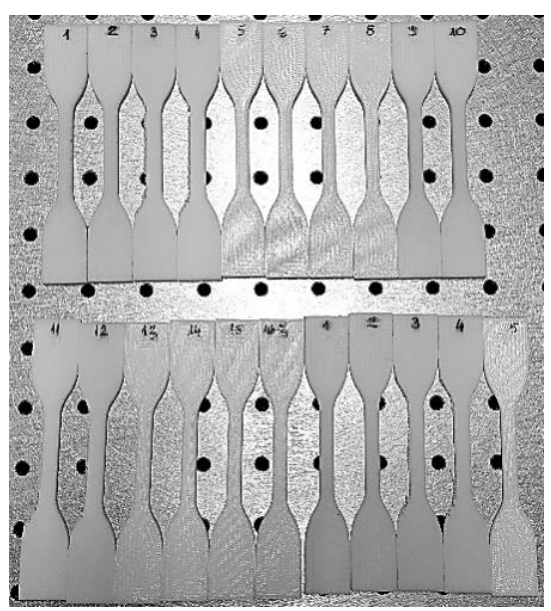

(a)
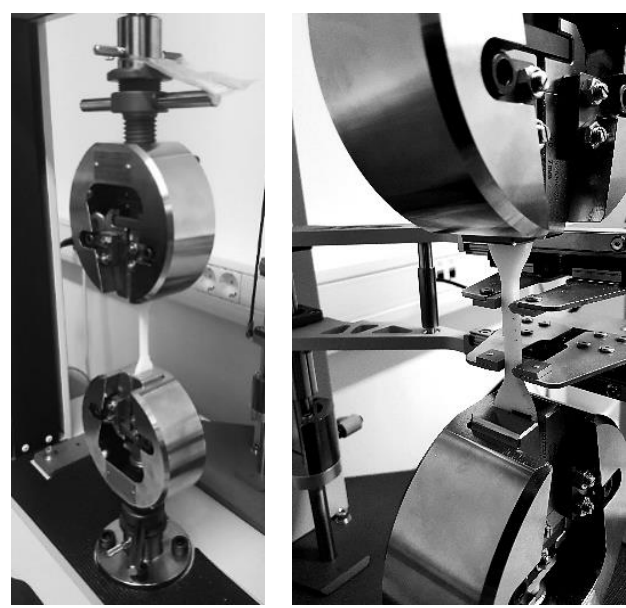

(b)

Fig. 5. Specimens used to test the properties of the ABS 3D printed material (a) and respective tensile tests (b) 
In order to optimize in this framework the 3D printing procedure, a thorough study of the influence of the orientation of the $3 \mathrm{D}$ components (horizontal or vertical, and at varying angles w.r.t. the motion axes of the printer) relating to printer's table is hence performed. The dimensions of the samples and the testing procedure are conducted in this frame according to the International Standard Test Method for Tensile Properties of Plastic ASTM D638 - 02a [20]. Eight samples are thus printed at various inclinations by using the "solid" (high-quality) printing modality; a second batch of eight samples is, in turn, printed by employing the "sparse" (lower quality) modality. Each of these 16 specimens is 115 $\mathrm{mm}$ long and $4 \mathrm{~mm}$ thick. Finally, a third batch of five samples is produced by using again the "solid" option, but this time the specimens are $2 \mathrm{~mm}$ thick (Figure 5a). The mechanical properties of the thus obtained ABS samples are assessed by employing the Shimadzu Autograph AGS-X micro-tensile testing machine [19] available again at the premises of the Laboratory for Precision Engineering and Micro- and Nanosystems Technologies of the Centre for Micro- and Nanosciences and Technologies in Rijeka [18] (Figure 5b).

The data obtained from the tensile tests for the $4 \mathrm{~mm}$ thick samples is shown in Figure 6. It can thus be concluded that the biggest elongations are, as expected, obtained for the samples that are positioned horizontally on printer's table (in the figure the samples positioned vertically are those that brake at elongations of ca. 1\%). Depending then on the angle of the specimens w.r.t. to the motion axes of the printer head, the results vary a little, but the difference is limited to ca. $5 \%$ of the obtained strength. In any case, the strength of the specimens printed with the "solid" printing modality (black lines in Figure 5) is generally up to 30\% higher than that of the samples attained by using the "sparse" option (grey lines in Figure 5). The "solid" option in the horizontal plane, allowing to achieve a strength of up to $35 \mathrm{MPa}$ and strains of up to ca. $1.8 \%$ is thus chosen for printing the components of the herein developed device.

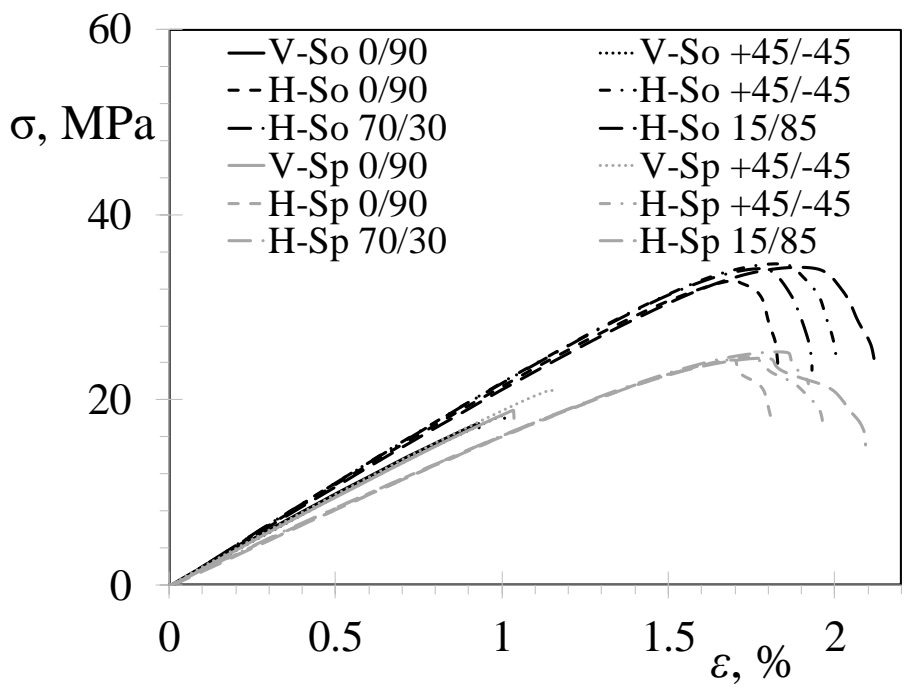

Fig. 6. Results of the tensile tests of the 3D printed specimens

Based on the performed tensile tests, the first mechanical components of the designed full arm mechatronics rehabilitation device, are therefore finally printed (Figure 7).

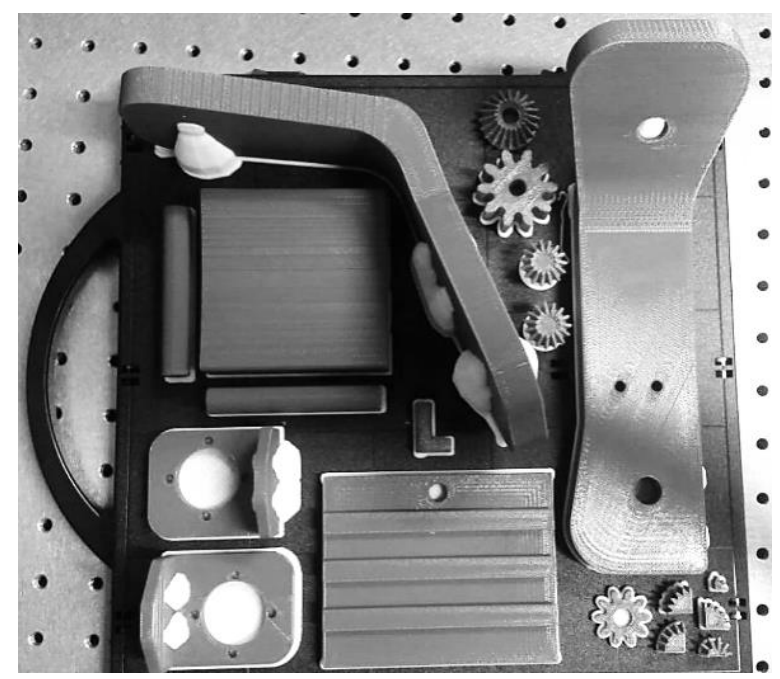

Fig. 7. First 3D printed mechanical components of the developed mechatronics arm rehabilitation device 


\section{Conclusions and outlook}

The design of the prototype of a full arm mechatronics rehabilitation device is described in this work. The study of the anatomy of human hand and of the state-of-the-art prototype devices is thus performed allowing to converge towards a list of requirements and the design guidelines for the herein created seven DOFs device. The 3D model of an innovative versatile, portable and cost-effective device is hence developed. Based on functional considerations, the corresponding actuating and sensing components are chosen and purchased. In parallel, the development of the control architecture based on the inverse kinematics of the overall device is also performed. Since one of the main objectives of the project is to accomplish a device that is as light as possible, the 3D printing technique is then chosen to produce the passive components of the device. Prior to printing the factual mechanical components, a campaign of tests aimed at determining the mechanical properties of the 3D printing material is thus also carried out on standardized samples. This all shows how technologically complex the project is, as it comprises the necessity of considering aspects related to machine design, to electrical engineering, to computing and programming with control theory and practise, but also to medicine.

In further work, electromyography (EMG) sensors will be integrated into system's architecture to allow an active control of the torque for each joint. This should enable controlling the value of the torque provided by the actuators so that only the supplemental torque needed by the patients for completing a certain activity is added. Also, a visual user interface in the form of "games" adaptable to the age and the motion capabilities of the single patients, and based on virtual and/or augmented reality, will be developed to enhance the motivation of the patients and their interactivity with the device. All of this should thus provide an advanced technological solution for the rehabilitation of patients for the Croatian hospitals and clinics, contributing hence to the community at large but also, implicitly, to achieving the public responsibility and accountability of the research and academic sector of Croatia.

An ongoing active communication with the Science and Technology Park (STeP Ri [21]) as well as the Technology Transfer Office of the University of Rijeka, Croatia [22], considering also the involvement of STeP Ri in the Enterprise Europe Network in the field of medicine [23], should also contribute to the rapid transfer of the developed device towards its concrete application.

\section{Acknowledgments}

The work described in this paper is enabled by the support of the "Moj ZABA Start" grant and by using the equipment funded via the ERDF project RC.2.2.06-0001 "Research Infrastructure for Campus-based Laboratories at the University of Rijeka - RISK”.

\section{References}

[1] https://www.stroke.org.uk/sites/default/files/the_burden_of_stroke_in_europe__challenges_for_policy_makers.pdf, (2017). King's College London for the Stroke Alliance for Europe (SAFE), The Burden of Stroke in Europe, Accessed on: 2018-05-02

[2] Nef, T.; Guidali, M. \& Riener R. (2009). ARMin III - Arm Therapy Exoskeleton with an Ergonomic Shoulder Actuation. Applied Bionics and Biomechanics, Vol. 6, No. 2, 127-142, ISSN: 1176-2322

[3] http://www.bridgecaresuites.com/stroke-rehabilitation-therapy-at-the-bridge-care-suites/, (2018). The Bridge Care Suites, Stroke Rehabilitation Therapy, Accessed on: 2018-04-04

[4] http://kbc-rijeka.hr/?lang=en, (2018). Clinical Hospital Centre Rijeka, Accessed on: 2018-05-03

[5] Gezgin, E. (2006). Biokinematic Analysis of Human Arm, M. Sc. Thesis, Graduate School of Engineering and Science, Izmir Institute of Technology, Izmir, Turkey

[6] Lenarčić, J. \& Stanišić M. (2003). A Humanoid Shoulder Complex and the Humeral Pointing Kinematics. IEEE Transaction on Robotics and Automation, Vol. 19, No. 3, 499-506, ISSN 1042-296X

[7] Schiele, A. \& van der Helm, F. C. T. (2006). Kinematic design to improve ergonomics in human machine interaction. IEEE Transaction on Neural Systems and Rehabilitation Engineering, Vol. 14, No. 4, 456-469, ISSN 1558-0210

[8] Gordon, C. C.; Churchill, T.; Clauser, C. E.; et. al. (1989). Anthropometric Survey of U.S. Personnel: Summary Statistics Interim Report for 1988, Identification code: ADA209600, Anthropology Research Project Inc., ISBN 978-989-758-225-7, Yellow Springs, OH, USA

[9] Krebs, H. I.; Ferraro, M.; Buerger, S. P; Hogan, N.; et. al. (2004). Rehabilitation robotics: Pilot trial of a spatial extension from MIT-Manus, Available from: https://jneuroengrehab.biomedcentral.com/articles/10.1186/17430003-1-5, Accessed: 2018-04-24

[10] http://www.sms.hest.ethz.ch/research/current-research-projects/armin-robot.html, (2018). ETH Zurich, ARMin Arm Rehabilitation Robotics, Accessed: 2018-05-03

[11] https://www.utwente.nl/en/et/be/research/projects/ar/, (2017). University of Twente, Robotic Aided Neuro rehabilitation of arm and hand functioning, Accessed on: 2018-04-24

[12] http://www.wearable-robotics.com/kinetek/, (2018). Kinetek Wearable Robotics, Accessed: 2018-05-07

[13] Carignan, C. \& Thang, J. (2007). A Configuration-Space Approach to Controling a Rehabilitation Arm Exoskeleton, Proceedings of the International Conference of Rehabilitation Robotics, Noordwijk, The Netherlands, ISSN: 19457898, ISBN: 978-1-4244-1319-5, DOI: 10.1109/ICORR.2007.4428425 
[14] Kucuk, S. \& Bingul Z. (2006). Robot Kinematics: Forward and Inverse Kinematics, In: Industrial Robotics - Theory, Modelling and Control, Cubero S. (Ed.), 117-148, InTechOpen, ISBN: 3-86611-285-8, Germany

[15] Spong, M. W.; Hutchinson, S. \& Vidyasagar, M. (2004). Robot Dynamics and Control - 2nd Ed., John Wiley and Sons, ISBN: 978-0-471-61243-8, New York, USA

[16] Asfour, T. \& Dillmann R. (2003). Human-like motion of a humanoid robot arm based on a closed-form solution of the inverse kinematics problem, Proceedings of the 2003 IEEE/RSJ International Conference on Intelligent Robots and Systems, Vol. 2, Las Vegas, Nevada, USA, ISBN: 0-7803-7860-1, pp. 1407-1412, IEEE, DOI: 10.1109/IROS.2003.1248841

[17] Munteanu, C. E.; Cismilianu, A.-M.; Chira, A.-I. \& Baran, D. (2017). Structural optimization of space components adapted for 3D printing, Proceedings of 8th DAAAM International Symposium on Intelligent Manufacturing and Automation, Zadar, Croatia, ISSN 1726-9679, ISBN 978-3-902734-07-5, Katalinic, B. (Ed.), pp. 1149-1157, DAAAM International, Vienna, Austria, DOI: 10.2507/26th.daaam.proceedings.162

[18] http://cmnzt.uniri.hr/, (2018). Centre For Micro- and Nanosciences and Technologies of the University of Rijeka, Croatia, Accessed on: 2018-04-25

[19] http://cmnzt.uniri.hr/lpemnt/wp-content/uploads/sites/2/2018/04/Katalog-LPEMNT_ENG.pdf, (2018). Centre for Micro- and Nanosciences and Technologies of the University of Rijeka, Croatia, Equipment Catalogue, Accessed on: 2018-04-25

[20] Standard Test Method for Tensile properties of Plastics, Designation: D638, ASTM International, USA, DOI: 10.1520/D0638-14, Available from: https://www.astm.org/Standards/D638.htm, Accessed on: 2018-04-30

[21] http://www.step.uniri.hr/, (2018). The Science and Technology Park of the University of Rijeka, Accessed on: 201805-02

[22] http://www.utt.uniri.hr, (2018). Technology Transfer Office of the University of Rijeka, Accessed on: 2018-30-04

[23] https://een.ec.europa.eu/about/sector-groups/healthcare, (2018). Enterprise Europe Network - Healthcare, Accessed on: $2018-05-03$ 